\title{
Trim State Discovery for an Adaptive Flight Planner
}

\author{
Guoxing $\mathrm{Yi}^{*}$ \\ Harbin Institute of Technology, Harbin, Heilongjiang, 150001, China \\ Ella M. Atkins. ${ }^{\dagger}$ \\ University of Michigan, Ann Arbor, Michigan, 48105, U.S.A
}

\begin{abstract}
Automatic trajectory planners require knowledge of an aircraft's flight envelope to ensure their solution is feasible. Existing flight management systems presume a nominal performance envelope, requiring online identification or characterization of any performance degradation. Given predictable failures such as a control surface jam, we can evaluate stability and controllability offline to establish a stablilizable trim state database that can be used in real-time to plan a feasible landing trajectory. In less-predictable cases, such as structural damage, performance can only be determined online. Given capable system identification and adaptive control capabilities, post-failure stability is achieved, and the attraction region can be safely explored in the neighborhood around the identified trim state. We propose a novel trim state discovery (TSD) strategy to automatically explore the operating envelope of an aircraft with appreciable but unknown damage or failures. We presume a system identification process identifies regions of attraction guaranteed locally, and that further damage or uncontrollable descent can result from excursion outside these regions. With the goal of identifying a sufficient flight envelope for landing, output from the TSD process feeds into an adaptive flight planner (AFP) that constructs safe landing flight plans as sequences of feasible trim states. We adopt a modified artificial potential field method to traverse the flight envelope space rather than physical space, incorporating envelope constraints as "obstacles" and desirable approach trim states as "attractors". An F-16 aileron jam scenario is presented to illustrate the utility of TSD for damage-resilient flight planning and guidance.
\end{abstract}

\section{Nomenclature}

$\begin{array}{ll}z & =\text { state vector } \\ V_{T} & =\text { flight velocity } \\ \alpha & =\text { attack angle } \\ \beta & =\text { sideslip angle } \\ p, q, r & =\text { body angle rate } \\ \mu & =\text { control vector } \\ x, y, z & =\text { aircraft's position } \\ \eta & =\text { configuration vector } \\ v & =\text { velocity vector } \\ \phi, \theta, \psi & =\text { Euler angles } \\ \gamma & =\text { flight path angle } \\ \dot{\psi} & =\text { turn rate }\end{array}$

\section{Introduction}

Millions of passengers and packages are transported by aircraft everyday. In 1997, there were 20.8 million departures and 43 million commercial flight hours world-wide. ${ }^{1}$ Aircraft safety has been a significant concern

\footnotetext{
${ }^{*}$ Associate Professor, Control Science \& Engineering Dept, Harbin Institute of Technology, Harbin, China.

${ }^{\dagger}$ Associate Professor, Aerospace Engineering Dept, University of Michigan, Ann Arbor, MI, Associate Fellow.
} 
of aircraft researchers and manufacturers. Extensive research has shown that flying is the safest form of commercial transportation. Even though aviation safety has improved from 1945 to 2004 by a factor of 200, the absolute number of fatalities in 2004 approximately matched the level of 1945 with 430 fatalities worldwide. ${ }^{2}$ Unmanned aircraft systems (UAS) are also challenged to operate safely, with 31 mishaps reported in 2008 within the United States Air Force. $^{3}$

Damage or failures that significantly impact performance introduce pressure to respond quickly and accurately. To improve the ability of pilots to guide a damaged aircraft to a safe landing, researchers have developed flight simulators for training pilots across a spectrum of possible damage and failure scenarios. ${ }^{4}$ In some cases, pilots have saved severely damaged aircraft through skill and judgment. But pilots also have been unable to recognize and recover from adverse conditions or in many cases have responded improperly, particularly under conditions of overload, stress, or fatigue. ${ }^{5}$ Accidents resulting from operator error continue to plague manned and unmanned aviation. ${ }^{6}$ Data from the UAS community indicates that from 21\% (Shadow) to $47 \%$ (Predator) of the accidents had contributing factors associated with human error. Within these incidents, 47\% (Hunter) to 68\% (Pioneer) are related to landing. ${ }^{\text {? }}$

To cope with in-flight emergencies, researchers have designed automation enhancements to help the pilot control the aircraft. Significant research effort has focused on novel control methods to recover stability with a damaged aircraft. ${ }^{8,9,10}$ Adaptive flight control methods adapt to system uncertainty caused by aircraft damage or failure, which includes aerodynamic changes, structural degradation, engine damage, and reduced flight control effectiveness. For example, Rysdyk et al proposed a neural network control system architecture, incorporating direct adaptive control with dynamic inversion. ${ }^{11}$ The simulation results of Kaneshige et al demonstrate that a neural flight control system can accommodate damage or failures over a range of failure conditions in a commercial aircraft. ${ }^{12}$ Nguyen and Krishnakumar developed a direct-indirect adaptive flight controller based on neural networks to maintain stability of a damaged aircraft. ${ }^{13}$ Page et al upgraded existing adaptive control laws and integrated them within an F/A-18C for flight testing. ${ }^{14}$ Results showed that the technique is effective for a variety of control surface jam conditions.

Although adaptive control methods maintain stability and controllability of a damaged aircraft, in many situations the flight envelope unavoidably contracts. The result may be the need for increased thrust or larger actuator deflections to compensate for the reduced performance. As saturation limits are reached, reference commands are no longer possible, necessitating alteration of the flight plan in addition to the control law. Aircraft dynamics are nonlinear, thus it is typically difficult to extend stability and controllability results in a manner that identifies the full operating envelope. In other words, although an adaptive controller can stabilize the aircraft, it typically cannot inform the pilots or autopilot whether the sequence of states they prefer for landing are feasible or not without further investigation.

Faced with in-flight damage/failure, pilots must be aware of compressed flight envelope bounds, not currently obtainable from cockpit displays. In addition, pilots must rapidly decide which runway should be selected as the landing site and what trajectory will properly reach this runway. To help the pilot make a valid sequence of decisions when damage/failure occurs, researchers have proposed an emergency flight management system. ${ }^{15,16}$ Chen and Pritchett propose an emergency flight planner that depends on predetermined plans and degraded dynamic models and examine its utility through pilot studies. ${ }^{15}$

In our previous research, an Emergency Flight Management Architecture has been proposed as illustrated in Figure 1. The Flight Plan Monitor continually validates the existing flight plan against the most current system model to verify feasibility of the flight plan. If the executing flight plan becomes infeasible, the pilot is notified via the Pilot Interface. Concurrently, the AFP is activated to generate a new flight plan. Within the AFP, a Landing Site Search (LSS) module identifies a safe landing site, currently defined as a runway deemed safe based on the degraded aircraft performance model. The Segmented Trajectory Planner then constructs a dynamically feasible trajectory to the landing site.

The Segmented Trajectory Planner relies on the feasible and stabilizable post-failure/damage trim flight states as building blocks of a segmented landing trajectory. In our previous work, the trim state database was calculated offline based on the damaged aircraft model. A stabilizable trimmed flight state database describes the feasible post-failure flight envelope, completely defining the performance characteristics of the aircraft after a specific failure. The database not only indicates the feasibility of each trim state but also defines the feasibility and characteristics of dynamic transition from one trim state to another. For failures we can characterize and classify (e.g., a control surface known to be jammed at a particular angle), we can evaluate the stability and controllability of any flight states to establish the stablilizable trim states database which should be used for trajectory planning. ${ }^{16-18}$ Unfortunately, many damage or failure cases that occur in flight are unknown due to our limited ability to directly sense or otherwise classify failure state. In these cases, we cannot recall and apply a pre-computed database of trim 
states guaranteed to be feasible. Instead, the aerodynamic characteristics of the damaged aircraft must be considered an uncertain nonlinear system, with each stabilizable trim state about an equilibrium point identified based on a locally-derived model.

This work introduces a Trim State Discovery (TSD) algorithm for an Adaptive Flight Planner (AFP). Without prior knowledge of the post-damage/failure flight envelope, TSD explores and exploits the feasible trim state space of the damaged aircraft. The goal of this process is to identify a sufficient, not comprehensive, trim state space to enable the AFP to build an emergency landing flight plan. Given nonlinear dynamics, we utilize the region of attraction around each stable trim state to guarantee stability is maintained during exploration. We adapt reactive motion planning methods to guide the exploration process, planning in "flight envelope space" rather than physical space. With this translation, we introduce an automated guidance protocol capable of conservatively targeting trim states to sequence for a safe landing.

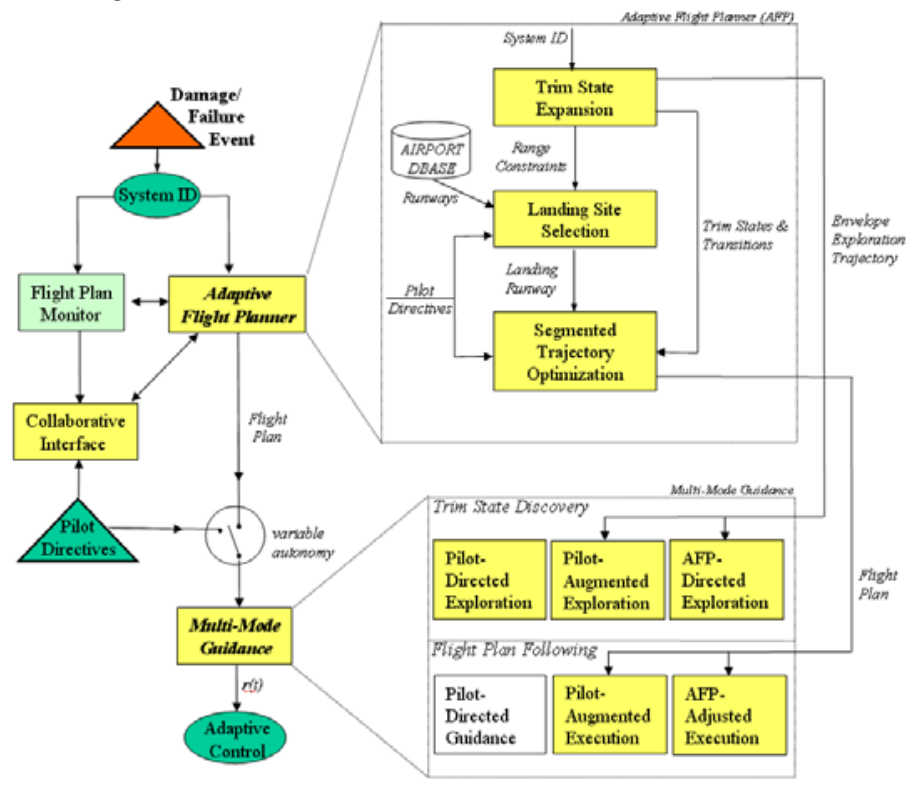

Figure 1. Emergency flight management architecture.

Below, we describe aircraft model preliminaries, followed by a motion planning review leading to definition of a Trim State Discovery (TSD) algorithm. A potential field path planning method was adapted to guide the TSD process, augmented with an edge-following algorithm to handle local minima. An F-16 aileron jam failure case study illustrates the effectiveness of TSD during post-failure flight planning. To avoid excessive exploration in 2D discovery, a 3D search process is presented and applied to the F-16 aileron jam failure scenario.

\section{Trim State}

A 6-DOF nonlinear aircraft model used to describe the aircraft equations of motion is given by

$$
f(\dot{z}, z, \mu)=0
$$

where $f$ is a vector of $n$ scalar nonlinear functions, and $z$ is the 12-dimensional state vector which includes the aircraft's position, attitude, angular velocities and linear velocities:

$$
z=\left[\begin{array}{llllllllllll}
V_{T} & \alpha & \beta & p & q & r & x & y & z & \phi & \theta & \psi
\end{array}\right]^{T}
$$

The control vector $\mu$ can be expressed as

$$
\mu=\left[\begin{array}{lllll}
\mu_{l t} & \mu_{r t} & \mu_{e} & \mu_{a} & \mu_{r}
\end{array}\right]^{T}
$$

with elevator (e), aileron ( $a$ ), and rudder $(r)$ control surface deflections and generalized left $(l t)$ and right $(r t)$ engine throttle terms used to enable asymmetric thrust application (if applicable). Vector $Z$ can be divided into two parts: aircraft configuration $\eta=\left[\begin{array}{llllll}x & y & z & \phi & \theta & \psi\end{array}\right]^{T}$ and velocity $v=\left[\begin{array}{lllll}V_{T} & \alpha & \beta & p & q\end{array}\right]^{T}$.

The trim state is a steady-state flight condition in which the aircraft velocities (linear and angular) will be constant over time. The trim state can be defined as

$$
\dot{v}^{*}=0
$$


The equation can be split as follows:

$$
\left(\dot{V}_{T}, \dot{\alpha}, \dot{\beta}\right) \equiv 0,(\dot{p}, \dot{q}, \dot{r}) \equiv 0,(\dot{\phi}, \dot{\theta}) \equiv 0, \dot{\psi} \equiv \dot{\psi}^{*}, \dot{h} \equiv \dot{h}^{*}
$$

where $\dot{\psi}^{*}$ and $\dot{h}^{*}$ represent the trim state turn rate and climb rate respectively. Since a trim state with constant turn rate and climb rate will be used as segments for trajectory planning, we define a reduced state vector

$$
\bar{Z}=\left[\begin{array}{llllllll}
V_{T} & \alpha & \beta & p & q & r & \phi & \theta
\end{array}\right]^{T}
$$

To determine trim state, a cost function is defined as

$$
J_{\text {trim }}(z, \mu)=\frac{1}{2} \dot{\bar{Z}}^{T} Q \dot{\bar{Z}}
$$

$z^{*}$, corresponding to $J_{\text {trim }}^{*}=0$, will be a trimmed or equilibrium flight state. Since this problem can not be solved analytically, numerical optimization has been employed. ${ }^{17-18}$ Additionally, the solution should be constrained as specified in Equations (8) and (9), where (9) indicates actuator saturation constraints with presumed-symmetric throttle $(t)$ scaled in the range [0 1$]$ and with control surface deflections measured in degrees.

$$
\begin{gathered}
h=h^{*}, v_{T}=v_{T}^{*}, \tan \theta=\frac{a b+\sin \psi^{*} \sqrt{a^{2}-\sin \gamma^{* 2}+b^{2}}}{a^{2}-\sin \gamma^{* 2}}, p=\dot{\psi}^{*} \sin \theta, q=\dot{\psi}^{*} \cos \theta \sin \phi, r=\dot{\psi}^{*} \cos \theta \cos \phi \\
\left|\mu_{t}\right| \leq 1,\left|\mu_{e}\right| \leq 25,\left|\mu_{a}\right| \leq 21.5,\left|\mu_{r}\right| \leq 30
\end{gathered}
$$

where $a=\cos \alpha \cos \beta, b=\sin \phi \sin \beta+\cos \phi \sin \alpha \cos \beta, \gamma^{*}$ is the trimmed flight path angle. Since the nonlinear system can be approximated by a linearization of its dynamics about a trim state in a small neighborhood surrounding that trim state, a linear perturbation method has been used to calculate the Jacobian matrices for each trim state. Therefore the stability or controllability will be evaluated by system eigenvalues and the controllability matrix. ${ }^{17-18}$ The stabilizable trim states will be used to compose segmented trajectories. Figure 2 shows the stable and controllable trim states of F-16 aircraft at altitude 2000 feet for a case in which the rudder was jammed at $20^{\circ}$. Figure 3 represents trim states of F-16 aircraft at altitude 2000 feet for a case in which the aileron was jammed at $5^{\circ}$. In the figures, a green asterisk indicates a naturally stable trim state with trimmed flight condition $\left(V_{T}, \dot{h}, \dot{\psi}\right)$, while a blue asterisk represents an unstable but controllable trim state. The unmarked trim states are infeasible or uncontrollable.

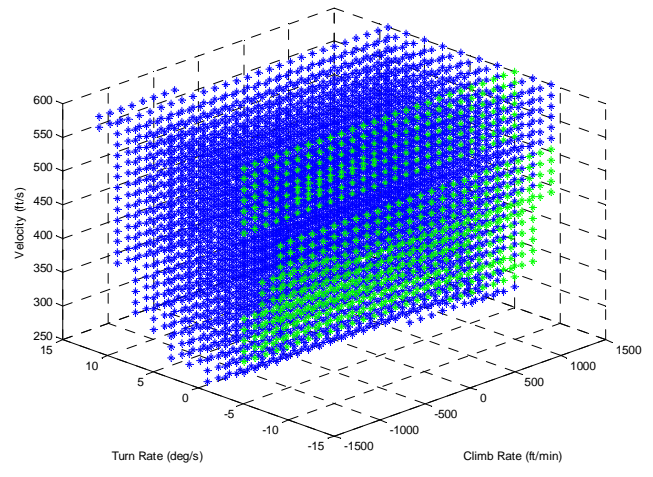

Figure 2. F-16 Rudder Jam Envelope. ${ }^{18}$

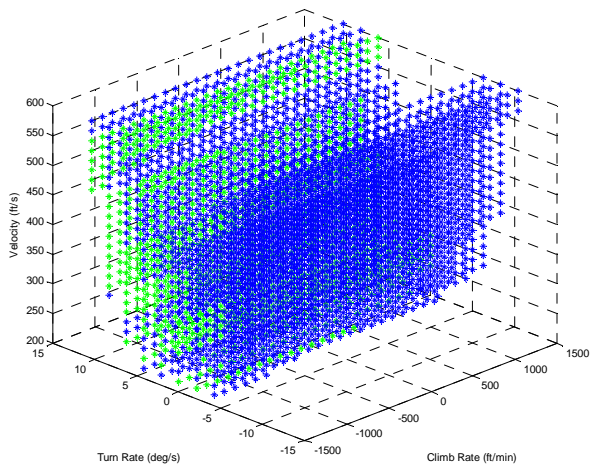

Figure 3. F-16 Aileron Jam Envelope. ${ }^{18}$

\section{Trim State Discovery (TSD)}

To cope with in-flight damage/failure, the AFP must guide the aircraft through a sequence of feasible trim states leading to a safe landing, in our work constrained to a published airport runway. Before transitioning from the current proven equilibrium state to a new state, the feasibility of the new state should be predicted - otherwise the aircraft may transition outside its operating envelope. In terms of dynamic system analysis, feasibility prediction translates to determining whether the new trim state belongs to the attraction region of the equilibrium state or not, and whether the new stability margin is sufficient for the damaged aircraft with a disturbance such as unexpected 
wind. The problem of determining the region of attraction of a stable equilibrium point for a nonlinear system has received substantial attention in the literature. Some researchers use a Lyapunov function method to estimate the attraction region of a stable equilibrium point in a nonlinear system. ${ }^{19,20}$ Chiang, Hirsch, and Wu present a comprehensive theory to derive a complete dynamical characterization of the stability boundary of a large class of nonlinear autonomous dynamical systems and proposed a method for finding the stability region based on its topological properties. ${ }^{21}$ Recently, linear matrix inequality (LMI) theory has been introduced to deal with nonconvex distance problems in attraction region estimation. ${ }^{22}$ Researchers have obtained a lower bound of the largest estimate of the domain of attraction for a fixed Quadratic Lyapunov function via LMI and have provided a condition for checking tightness of a lower bound.. Tibken used real algebraic geometry theory to compute subsets of the region of attraction of asymptotically stable stationary points of polynomial systems by reformulating the problem as a LMI. ${ }^{23}$ Amato, Cosentino, and Merola proposed a method to investigate the attraction region of equilibrium points of quadratic systems, ascertaining whether a certain box belongs to the attraction region. ${ }^{24}$ In our AFP research, we focus on the process of final approach trim state identification and sequencing and flight envelope exploration. We presume the properties of the flight state within the certain neighborhood of present trim state can be obtained rather than focusing on specifics of attraction region estimation.

In our work, the process of trim state discovery is based on an algorithm adapted from robot motion planning with obstacle avoidance. Instead of traveling through physical (3-D) space, our planner computes a path trim-state space with 3-D coordinates $\left(V_{T}, \dot{h}, \dot{\psi}\right)$, translated to physical 3-D space to verify required altitude and airspace

constraints are met. We further presume, based on our past experience with damage and failure models, the trim states achievable at high altitudes will also be achievable at lower altitudes. This assumption implies each identified trim state will remain feasible at or below the maximum altitude at which stable operation at this state was demonstrated.

Motion planning can be categorized as static, in which all obstacle information is known prior to planning, or dynamic, in which environment (obstacle) information becomes known to the planner only through real-time sensing of its local environment. ${ }^{25}$ In our research, trim state discovery is used to characterize unknown failures, for which envelope constraint information cannot be obtained prior to the discovery process. This requires use of a dynamic path planning strategy in which envelope constraints, as they are approached, are modeled as "obstacles" for the trim-state-space path planner. Many researchers have studied dynamic methods of robot path planning with obstacle avoidance. Lumelsky proposed the Bug algorithms (Bug 1 \& Bug 2) to solve the dynamic obstacle avoidance problem. ${ }^{26,27}$ With a Bug strategy, a robotic vehicle will move directly towards its goal state unless an obstacle is found, in which case the robot will travel around the obstacle boundary until no known obstacle is present between the direct path toward the target. This strategy is not optimal but is intuitive when only local sensing is available, as might be the case in a system that only has knowledge of its local region of attraction. An artificial potential field (APF) strategy is a popular alternative approach to dynamic path planning. ${ }^{28,29}$ With APF a vehicle follows the gradient of the cumulative potential field modeling the goal/target with attractive potential and obstacles with repulsive potentials. Borenstein and Korem introduced the real-time vector field histogram method to lead the robot a region with minimum obstacle density. ${ }^{30,31}$ Rickert, Brock, and Knoll presented experimental results of adaptive balancing of exploration an exploitation in the APF context, which can significantly improve planner performance. ${ }^{32}$

We adopt an APF dynamic path planning method to autonomously guide the trim state discovery process. Without loss of generality, we presume the aircraft establishes and holds a stable initial trim state just after the failure/damage occurs. For safety, the pilot or adaptive flight management system must adjust the aircraft flight plan to follow a sequence of feasible flight states to landing. Because the failure is initially unknown, however, neither crew nor autopilot can be confident a nominal landing trajectory will be possible, even for the next flight condition to which they intend to transition. The baseline purpose of trim state discovery is to enable the pilot/autopilot to confirm the stability of a nominal final approach trim state sequence, including transitions. If the nominal sequence is not feasible, then the purpose of trim state discovery becomes to identify an off-nominal stable envelope sufficient for building an off-nominal but safe landing trajectory. Given the existence of unknown, nonlinear aircraft dynamics, the stability of a trim state cannot be evaluated until the damaged aircraft transitions to a local neighborhood of that state. We can then describe the problem of trim state discovery (TSD), as follows:

Given an initial stable damaged aircraft "position" in trim state-space $s_{0}=\left(V_{0} \dot{\psi}_{0} \gamma_{0}\right)^{T}$ and an ideal final approach trim state $s_{\text {app }}$, generate a continuous path $T$ in trim state-space from $s_{0}$ to $s_{\text {app }}$, where $T$ is a sequence of continuous trim states and transitions. All trim states in $T$ must be stablilizable in the presence of disturbances. 
Edges of flight envelope, defined by actuator saturation or stabilizability constraints, are considered obstacles on the path to the goal state $s_{a p p}$. These obstacles are considered impenetrable. Figure 4 depicts a path through (flight path angle - turn rate) space from initial state $s_{0}$ to $s_{a p p}$. In this illustration there are multiple flight envelope boundaries that must be circumvented.

With the above formulation, trim state discovery is mapped to motion planning with obstacle avoidance. Every step (local transition) through trim-state space involves two phases: exploration and exploitation. Exploration seeks to understand of the neighborhood around the present trim state (equilibrium point), without respect to state discovery. The exploration

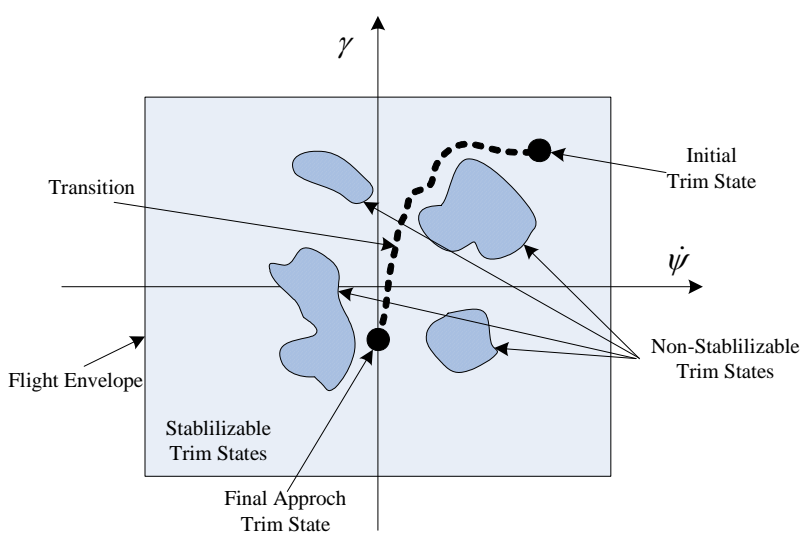

Figure 4. Trim State Discovery (TSD). process is to determine the local region of attraction to determine local region stability. Exploitation relies on this characterization to identify an appropriate next trim state given the designated goal and obstacles identified through the current or previous exploration analyses.

Figure 5 illustrates the State Discovery (TSD) algorithm. First, the pilot or autopilot must establish and maintain a stable trim state after the failure of damage event. This state is the initial trim state $s_{0}=\left(V_{0} \dot{\psi}_{0} \gamma_{0}\right)^{T}$. In step 3, transition time sequence $t_{\text {trans }}$ is determined to have three time periods. During the process of discovery, the aircraft maintains its present state for $t_{s h 1}$, follows a linearly-approximated transition from $s_{i}$ to $s_{i+1}$ during $\left[t_{s h 1}, t_{s h 1}+t_{s}\right]$, and then maintain $s_{i+1}$ for $t_{s h 2} \cdot t_{s h 1}$ and $t_{s h 2}$ are a function of system identification convergence time, which is in turn a function of dynamic settling time. In our simulation, both $t_{s h 1}$ and $t_{s h 2}$ were presumed $5 \mathrm{~s}$. $t_{s}$ values are a function of control system rise and settling time over the transition, which for unknown damage must be adapted based on observed system response.

As TSD explores trim state-space, the aircraft also flies through physical space. TSD must therefore determine whether the altitude is sufficiently high for exploration to continue with zero or negative flight path angle appropriate to subsequently guide the aircraft to landing. If altitude is insufficient, TSD must direct the aircraft to a feasible stable state with positive flight path angle to regain sufficient altitude for continued TSD. The TSD process is then continued once a sufficient altitude is reached. All discovered feasible trim states will be saved as well as the attraction region of each trim state. Once TSD finds a solution, the Adaptive Flight Planner will plan a safe trajectory for the aircraft landing, using the explored trim state database.

For efficient exploration, the TSD must incrementally direct the aircraft through a series of trim state-space transitions connecting the initial trim state to the targeted final approach trim state set. As indicated above, we adopt a modified artificial potential field method to determine the direction and length of each progressive step through trim state-space. We model target state $s_{\text {app }}$ with a positive (attractive) potential and the obstacles as regions with a negative (repulsive) potential. Let $\vec{F}_{A}$ represent the attractive potential on $s_{t}$ due to goal $s_{a p p}$, and let the obstacle apply repulsive force $\vec{F}_{P}$ on $s_{t}$. These two forces will generate resultant force $\vec{F}_{R}$. As a physical particle analogue, the vehicle is then guided to follow this artificial potential field via a transition in direction $\vec{F}_{R}$. For each transition iteration, step length depends on the magnitude of $\vec{F}_{R}$ and the local attraction region. Figure 6 shows how the three forces interact. The calculation of the forces is given by:

(1) Attractive force:

$$
\vec{F}_{A}=F_{A 0}\left(1-\exp \left(\frac{\left|\vec{S}_{a p p}-\vec{s}_{t}\right|}{\left|\vec{S}_{a p p}-\vec{S}_{0}\right|}\right)\right) \frac{\vec{S}_{a p p}-\vec{s}_{t}}{\left|\vec{S}_{a p p}-\vec{S}_{c}\right|}
$$




\section{Algorithm DISCOVERY $\left(s_{0}, T_{0}, t_{0}\right)$}

1. Set initial state $s_{0}=\left(V_{0}, \dot{\psi}_{0}, \gamma_{0}\right)^{T}$ with $\mu_{0}=\left(\mu_{t_{0}}, \mu_{r_{0}}, \mu_{e_{0}}, \mu_{a_{0}}, \mu_{r_{0}}\right)^{T}$

2. Read initial position $T_{0}=\left(x_{0}, y_{0}, h_{0}, t_{0}\right)^{T}$

3. Determine transition time sequence $t_{\text {trans }}=\left(t_{\text {sh } 1}, t_{s}, t_{\text {sh } 2}\right)^{T}$

4. Set final approach trim state $s_{\text {des }}=\left(V_{\text {des }}, \dot{\psi}_{\text {des }}, \gamma_{\text {des }}\right)^{T}$

5. while (final trim state not be discovered)

6. Execute Trim State Discovery $s_{i}=\left(V_{i} \dot{\psi}_{i} \gamma_{i}\right)^{T} \rightarrow s_{i+1}=\left(V_{i+1} \dot{\psi}_{i+1} \gamma_{i+1}\right)^{T}$

7. Update Trim States Sequence $S=\left\{s_{j}\right\}_{j=0}^{i} \cup\left\{s_{i+1}\right\}$ and region of attraction of every explored trim state $A=\left\{a_{j}\right\}_{j=0}^{i} \cup\left\{a_{i+1}\right\}$

8. Compute trajectory $T_{i+1}=\left(x_{i+1}, y_{i+1}, h_{i+1}, t_{i}\right)^{T}=\operatorname{trajectory}\left(T_{i}, s_{i}, s_{i+1}, t_{\text {trans }}\right)$

9. Compute the estimate time of Trim State Discovery: $t_{T S D}=\operatorname{time}\left(s_{i+1}, s_{\text {des }}, T_{\text {trans }}\right)$

10. Compute the estimate lost of altitude: $\Delta h_{l}=\operatorname{altitude}\left(s_{i+1}, s_{\text {des }}, t_{T S D}\right)$

11. Compute minimum altitude $h_{\min }$ for path planning after trim state discovery

12. if $h_{0}-h_{l}<h_{\min }$

13. Estimate appropriate altitude for state $s_{i+1}: h_{a p p}^{i+1}=\operatorname{calaltitude}\left(s_{i+1}, s_{\text {des }}, h_{\min }, h_{i+1}\right)$

14. if exist $s_{k}=\left(V_{k} \dot{\psi}_{k} \gamma_{k}\right)^{T}$ with $\gamma_{k}>0$ (Search in $S=\left\{s_{j}\right\}_{j=0}^{i+1}$ )

15. Transit state to $s_{k}$ and compute trajectory $T_{i+1}^{n+1}=\operatorname{trajectory}\left(T_{i+1}^{n}, s_{k}, s_{i+1}, t_{\text {trans }}\right)$

16. While $h_{i+1}^{n+1}<h_{\text {app }}^{i+1}$

17. Estimate altitude while back to $s_{i+1}: h_{\text {est }}^{i+1}=\operatorname{estialtitude}\left(s_{k}, s_{i+1}, h_{i+1}^{n+1}\right)$

18. Hold $s_{k}$ and compute trajectory $T_{i+1}^{n+1}=\operatorname{trajectroy}\left(T_{i+1}^{n}, s_{k}, t_{\text {trans }}\right)$

19. End while

20. else

21. Discover to a new trim state $s_{\text {new }}$ with positive path angle

22. Compute trajectory $T_{i+1}^{n+1}=\operatorname{trajectory}\left(T_{i+1}^{n}, s_{\text {new }}, s_{i+1}, t_{\text {trans }}\right)$

23. While $h_{i+1}^{n+1}<h_{a p p}^{i+1}$

24. Estimate altitude while transit to $s_{i+1}, h_{\text {est }}^{i+1}=\operatorname{estialtitude}\left(s_{\text {new }}, s_{i+1}, h_{i+1}^{n+1}\right)$

25. Hold $s_{\text {new }}$ and compute trajectory $T_{i+1}^{n+1}=\operatorname{trajectory}\left(T_{i+1}^{n}, s_{\text {new }}, t_{\text {trans }}\right)$

26. End while

27. End if

28. Transit back to trim state $s_{i+1}$

29. End if

30. End while

31. Compute trim state sequence that should be explored around $s_{d e s}, S_{e p l}=\left\{s_{e p l}^{j}\right\}_{j=1}^{n}$

32. Explore $S_{e p l}$ and save new trim states $S=\left\{s_{j}\right\}_{j=0}^{i} \cup\left\{s_{i+1}\right\}, s_{i+1}=s_{e p l}^{j}$

33. Compute trajectory $T_{i+1}=\left(x_{i+1}, y_{i+1}, z_{i+1}\right)^{T}=\operatorname{trajectory}\left(T_{i}, s_{i}, s_{i+1}, t_{\text {trans }}\right)$

Figure 5. Trim State Discovery Algorithm. 
(2) Centroid of the detected obstacle:

$$
\vec{s}_{c}^{i}=\left(x_{c}^{i}, y_{c}^{i}\right)=\left(\frac{1}{A_{0}^{i}} \int_{\text {Area }} x d A^{i}, \frac{1}{A_{o}^{i}} \int_{\text {Area }} y d A^{i}\right)
$$

(3) Repulsive force:

$$
\vec{F}_{P}=F_{p 0} \sum_{i=1}^{n}\left(\frac{A_{o}^{i}}{A_{a}}\right) \cdot \frac{\vec{s}_{t}-\vec{s}_{c}^{i}}{\left|\vec{s}_{t}-\vec{s}_{c}^{i}\right|}
$$

(4) Resultant force:

$$
\vec{F}_{R}=\vec{F}_{P}+\vec{F}_{A}
$$

where $\vec{s}_{0}, \vec{s}_{t}$ and $\vec{s}_{c}^{i}$ are position vectors of target state, present state and the $i$ th dynamically-discovered (explored) obstacle centroid respectively. $A_{o}^{i}$ and $A_{a}$ are the area of the ith explored obstacle and local attraction region respectively. $F_{A 0}$ and $F_{p 0}$ are the adjustable coefficients of force which will be modified during debugging. The TSD will follow a sequence of transitions to the target final approach trim state with obstacle avoidance, under the action of the resultant force. However the simple potential field method has many shortcomings, especially the sensitivity to local minima that usually arise due to the symmetry of the environment and due to concave obstacles or multiple obstacles that together result in a concave region of cumulative potential. Figure 7 illustrates a case in which a local minimum is present. To cope with local minima, we adopt an edge-following algorithm which will explore the obstacle along its boundary until the goal can again be pursued. In trim state-space, this corresponds to traversing within but near the local edge of the flight envelope with respect to primary state-space parameters flight path angle and turn rate. In our APF algorithm, a local minimum is detected by monitoring the speed of state transition. The average speed of the latest ten transitions will be approximately zero when the system becomes trapped. After the trap is detected, the TSD will transition along the obstacle in a direction perpendicular to the repulsive force. The direction is related with the path direction, as follows:

$$
\overrightarrow{\text { step }}_{i}=\left\{\begin{array}{l}
\text { steplength } \cdot \frac{\vec{A}}{|\vec{A}|} \quad \text { if }\left(\vec{s}_{i}-\vec{s}_{i-1}\right) \cdot \vec{A} \geq 0 \\
\text { steplength } \cdot \frac{\vec{B}}{|\vec{B}|} \quad \text { if }\left(\vec{s}_{i}-\vec{s}_{i-1}\right) \cdot \vec{A}<0
\end{array}\right.
$$

where $\vec{A}$ and $\vec{B}$ are vectors perpendicular to repulsive force $\vec{F}_{P}$. When the exploitation activity is in edge-following mode, the distance between the edge and the present trim state guides the transitions through trim state space. If the distance is too far, the TSD may diverge away from the edge. Because of the limited distance to which attraction region can be safely projected, the TSD may return to the original local minimum then repeat the same exploration steps, again trapped. If the current state approaches too close to the obstacle (flight envelope boundary), the trim state may lose robustness to disturbances such as wind and imprecisely-computed model parameters. An additional repulsive force is introduced to deal with this problem, as seen in the following equation:

$$
\overrightarrow{\text { step }}_{i}= \begin{cases}\overline{\operatorname{step}}_{i}+0.2 \cdot \vec{F}_{P} & \text { if } A_{o} / A_{e}>\delta_{1} \\ \overline{s t e p}_{i}-0.2 \cdot \vec{F}_{P} & \text { if } A_{o} / A_{e}<\delta_{2} \\ \overline{s t e p}_{i} & \text { if } \delta_{2} \leq A_{0} / A_{e} \leq \delta_{1}\end{cases}
$$


where $\delta_{1}$ and $\delta_{2}$ are the coefficients defining the close degree of $P_{t}$ and the obstacle, which will also be selected in debugging. In our simulation, $\delta_{1}$ equals 0.75 , and $\delta_{2}$ equals 0.25 . Once the obstacle is no longer introducing a local minimum, the TSD resumes its nominal algorithm to follow a path to the target trim state region. We use two constraints to determine whether the obstacle has been passed: $\theta_{3} \leq 15^{\circ}$ and $D_{t} \leq D_{0}$, where $\theta_{3}$ is the angle between $\vec{F}_{A}$ and $\vec{F}_{P}, D_{t}$ is the distance between the target state and present state, and $D_{0}$ is the distance between the target and the local minimum. If TSD finds an infeasible final approach state with the given velocity, TSD will change the airspeed within nominal performance interval $\left[v_{\min }, v_{\max }\right]$ to seek further envelope exploration. Figures 8 and 9 illustrate the process of TSD in complex obstacle environments.

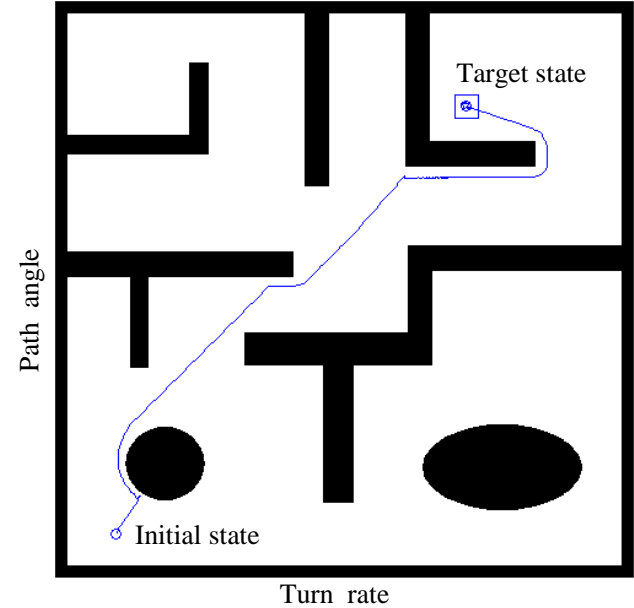

Figure 8: Dynamic Path Planning I

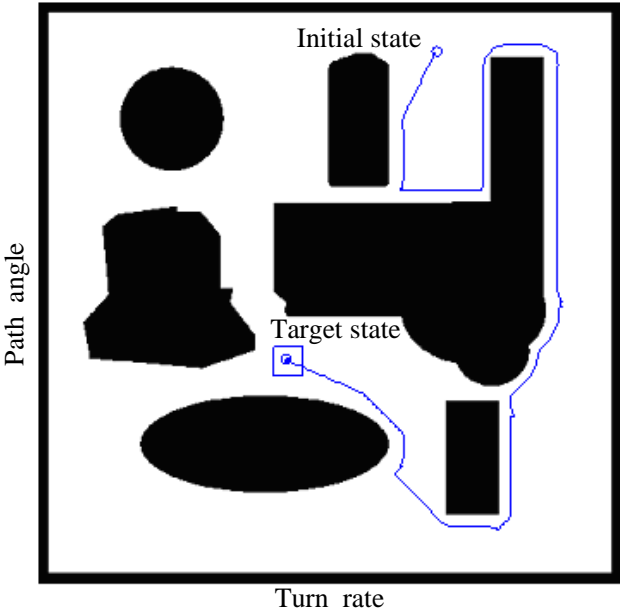

Figure 9: Dynamic Path Planning II

When the final approach trim state is feasible, the Trim State Discovery (TSD) process is able to guide the aircraft to this state in trim state-space. When the neighborhood of the targeted approach state is reached, this neighborhood is explored to more closely examine the ability of the aircraft to robustly operate in this region of trim state space. If the stability margin is not sufficient, TSD will change some condition, such as path angle, and explore again. Figure 10 shows the process of exploration.

Steering by our modified artificial potential field method, the exploitation process can escape local minima, not possible using the traditional potential field method only. However there may be a closed region in the 2-D turnrate/path-angle flight envelope for some damage or failure cases. Figure 11 shows such a situation. When the system is in edge-following mode, TSD initially determines the transition was caught in a closed trap if the state returned to a previously-identified local minimum. In addition, the edge of the local obstacle will be explored in the edge-following process.

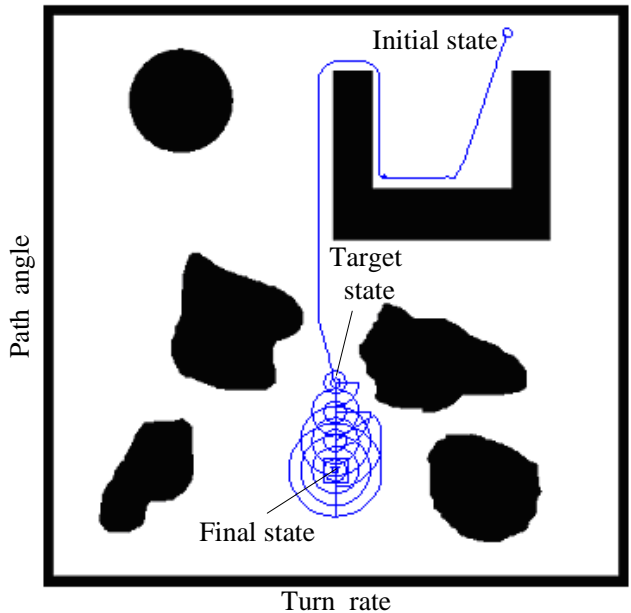

Figure 10: Neighborhood Exploration

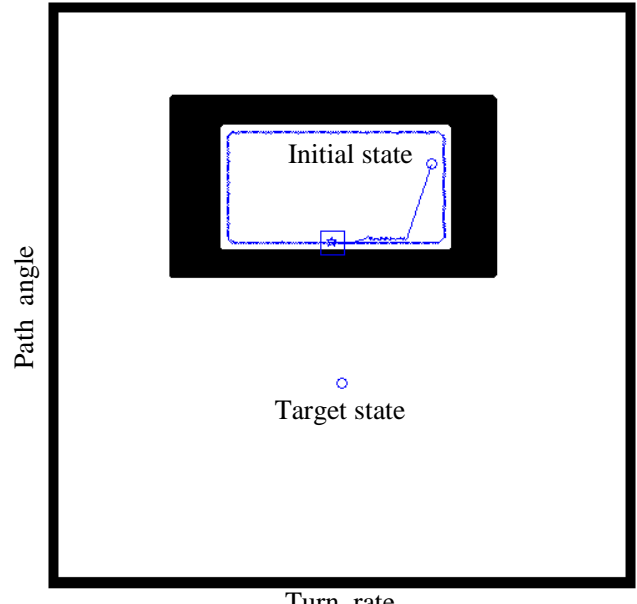

Figure 11: Target unreachable with Closed Obstacle

9

American Institute of Aeronautics and Astronautics 


\section{Case Study for 2D Trim State Discovery}

Our Trim State Discovery (TSD) algorithm was applied to a damaged F-16 aircraft with aileron jammed at -10 degrees. Figure 12 shows the flight envelope of the damaged F-16 aircraft. The color at each trim state indicates its characteristics, with green indicating a stable and controllable trim state, while blue represents an unstable but controllable trim state. The initial trim state is defined as: $h=1000 \mathrm{ft}, V_{T}=400 \mathrm{ft} / \mathrm{s}, \dot{\psi}=16.5^{\circ}$ and $\gamma=-1^{\circ}$. The final (targeted) trim state is defined as: $\dot{\psi}=0$ and $\gamma=-3^{\circ}$. TSD explored the two-dimensional trim state space, with turn rates from $-20^{\circ} \sim+20^{\circ}$ and flight path angles from $-10^{\circ} \sim+10^{\circ}$. Note that the envelope characteristics can be exactly obtained if the state belongs to a certain neighborhood of the present trim state, $\pm 0.2^{\circ}$ in the path angle dimension and $\pm 0.4^{\circ}$ in the turn rate dimension. Figures 13 to 17 show the process of two dimensional Trim State Discovery. When velocity is changed from $400 \mathrm{ft} / \mathrm{s}$ to $275 \mathrm{ft} / \mathrm{s}$, TSD is caught in a closed trap thus could not steer to the final approach trim state. In this case the velocity must also be changed. For simplicity, TSD kept path angle and turn rate constant during velocity excursions to maintain the continuity of the exploration and exploitation process. Figure 18 represents the full path of TSD with different conditions. Table 1 lists the corresponding numerical data.

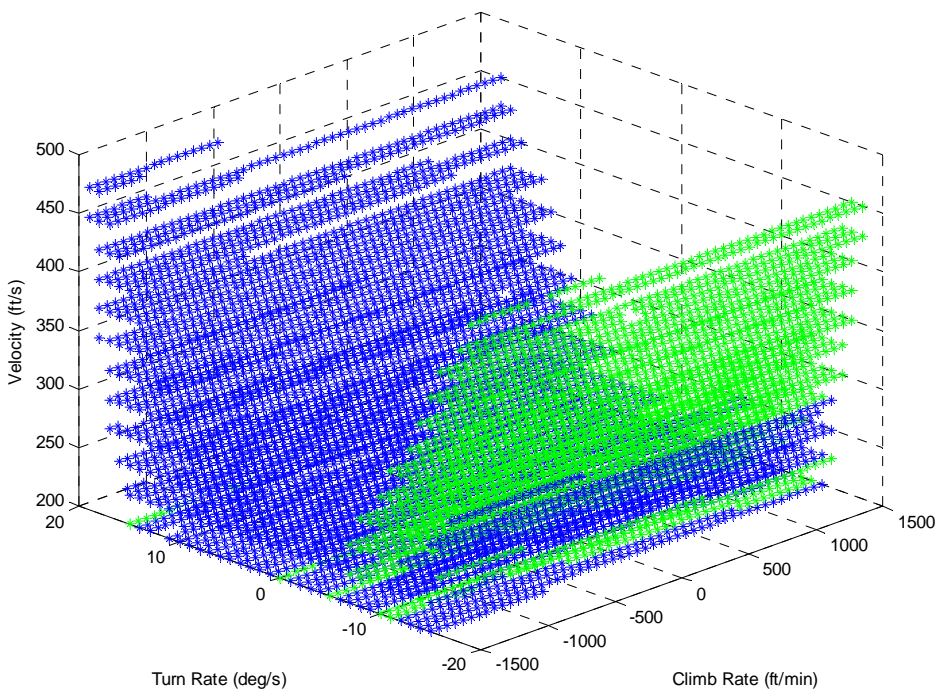

Figure 12: Trim States of Damaged F-16 Aircraft

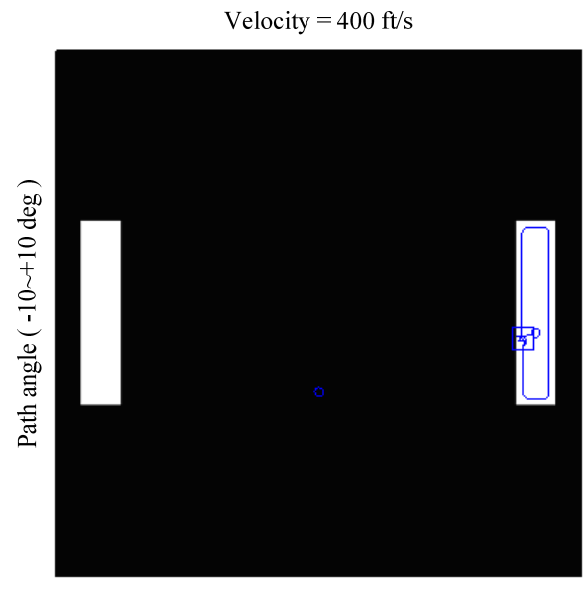

Turn rate $(-25 \sim+25 \mathrm{deg})$

Figure 13: Trim State Discovery I

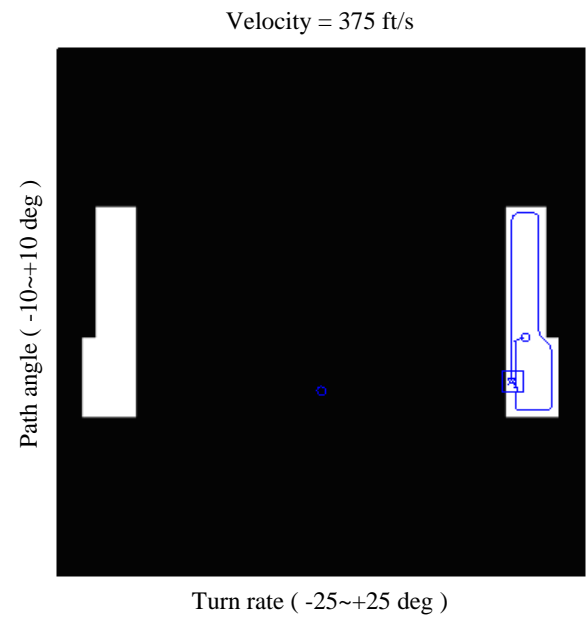

Figure 14: Trim State Discovery II 


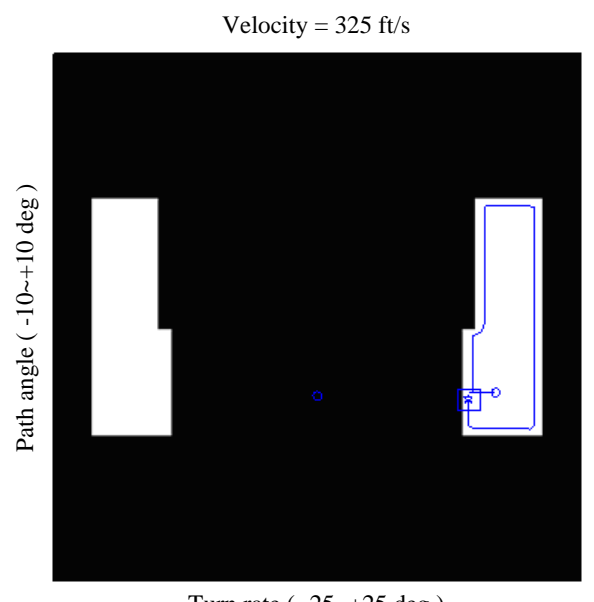

Turn rate ( $-25 \sim+25$ deg )

Figure 15: Trim State Discovery III

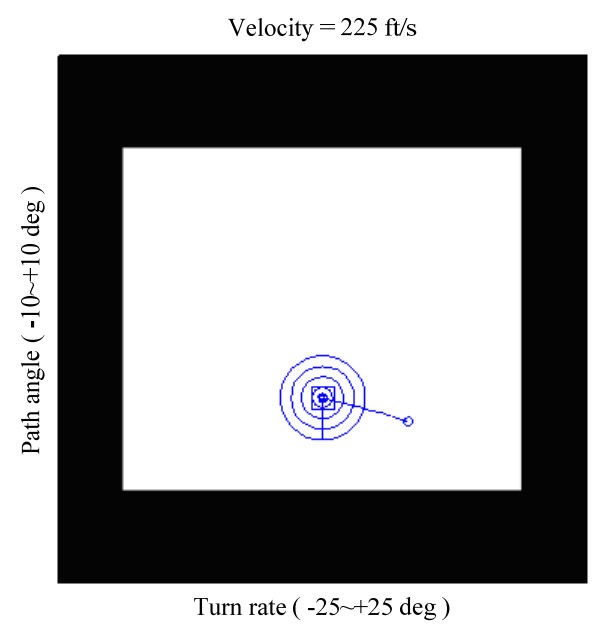

Figure 17: Trim State Discovery V

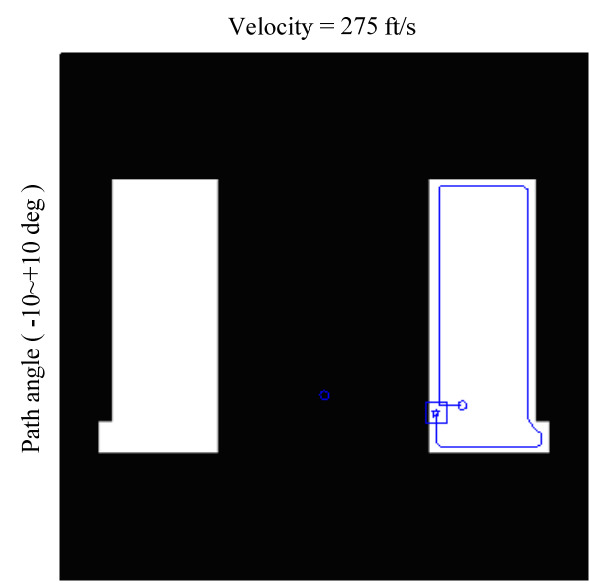

Turn rate $(-25 \sim+25 \mathrm{deg})$

Figure 16: Trim State Discovery IV

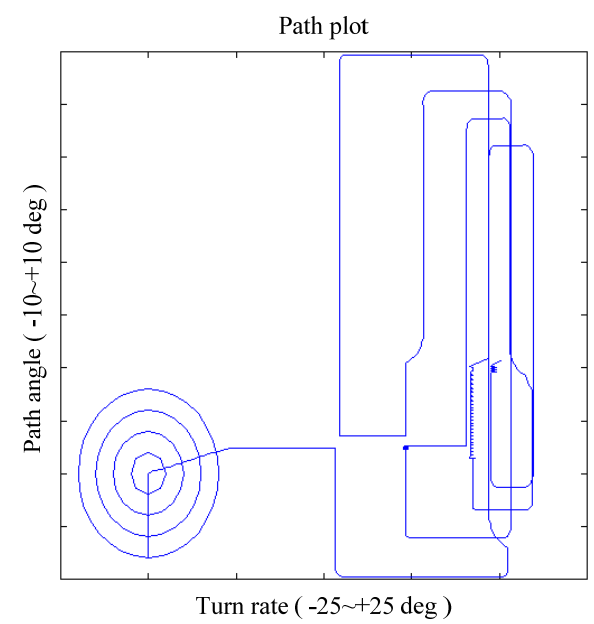

Figure 18: Discovery Path

Table 1. Exploration results.

\begin{tabular}{c|c|c|c|c|c}
\hline$V_{T}$ & $400 \mathrm{ft} / \mathrm{s}$ & $375 \mathrm{ft} / \mathrm{s}$ & $325 \mathrm{ft} / \mathrm{s}$ & $275 \mathrm{ft} / \mathrm{s}$ & $225 \mathrm{ft} / \mathrm{s}$ \\
\hline$\gamma_{\min }$ & $-3.7^{\circ}$ & $-3.9^{\circ}$ & $-4.5^{\circ}$ & $-5.3^{\circ}$ & $-1.0^{\circ}$ \\
\hline$\gamma_{\max }$ & $+3.7^{\circ}$ & $+3.9^{\circ}$ & $+4.5^{\circ}$ & $+5.3^{\circ}$ & $+5.0^{\circ}$ \\
\hline$\dot{\psi}_{\min }$ & $+15.0^{\circ}$ & $+14.0^{\circ}$ & $+11.0^{\circ}$ & $+8.0^{\circ}$ & $-4.0^{\circ}$ \\
\hline$\dot{\psi}_{\max }$ & $+18.0^{\circ}$ & $+18.0^{\circ}$ & $+17.0^{\circ}$ & $+17.0^{\circ}$ & $+4.0^{\circ}$ \\
\hline
\end{tabular}

\section{3D Trim State Discovery}

The above two dimensional Trim State Discovery (TSD) algorithm and results suggest including velocity as well as turn rate and path angle in the TSD search. We again utilize a potential field adaptation to 3-D trim state-space path planning. Figure 15 presents the composition of forces in three dimensional space. The symbols have the same definitions with Figure 6, with $s_{c}$ representing the centroid of the intersection block of an obstacle and the explored region. Three dimensional Trim State Discovery (TSD) will guide the exploration process through changing $V_{T}, \dot{\psi}$ and $\gamma$ to final approach trim state with obstacle avoidance. By using three dimensional algorithm, the repetitive search through path angle - turn rate from the two dimensional case can also be resolved. Therefore, the time of TSD could be significantly reduced, improving the responsiveness of a damaged aircraft to determine a sufficient envelope for landing. 
The damaged F-16 model with aileron jammed at -10 degrees was also used in three dimensional TSD simulations. The final trim state is specified as: $\dot{\psi}=0$ and $\gamma=-3^{\circ}$, with velocity to be determined during exploration. The initial trim state is defined as $h=10000 \mathrm{ft}, V_{T}=400 \mathrm{ft} / \mathrm{s}, \dot{\psi}=15.5^{\circ}$ and $\gamma=2.9^{\circ}$ for the first simulation and $h=10000 \mathrm{ft}, V_{T}=450 \mathrm{ft} / \mathrm{s}, \dot{\psi}=-16^{\circ}$ and $\gamma=-1.3^{\circ}$ for the second simulation. Figure 16 and Figure 17 show the results of three dimensional Trim State Discovery (TSD). The green dots

indicate stablizable trim states, and blue dots and line present discovery path of TSD. Compared with two dimensional

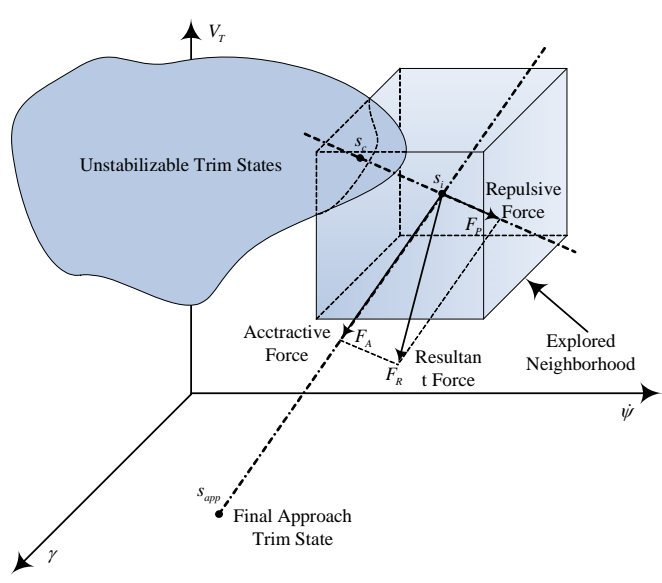

Figure 15: Potential Force in 3D cases, three dimensional TSD has higher efficiency and fewer transitions. The repetition in exploration process was averted.

Figure 18-21 present the effect on flight trajectory by different $t_{\text {rans }}$. Transition time, $t_{\text {rans }}$ is used to indicate the transition time between the adjacent trim states in the path of TSD. For the first case of 3D Trim State Discovery(TSD), we process simulations with different $t_{\text {rans }}$. Figures 18-21show the trajectories when $t_{\text {rans }}$ equals $5 \mathrm{~s}$, 10s, 20s and 40s respectively. These trajectories will be used as the initial condition of the landing path planner. The figures show that trajectories are highly influenced by trim state hold and transition times.

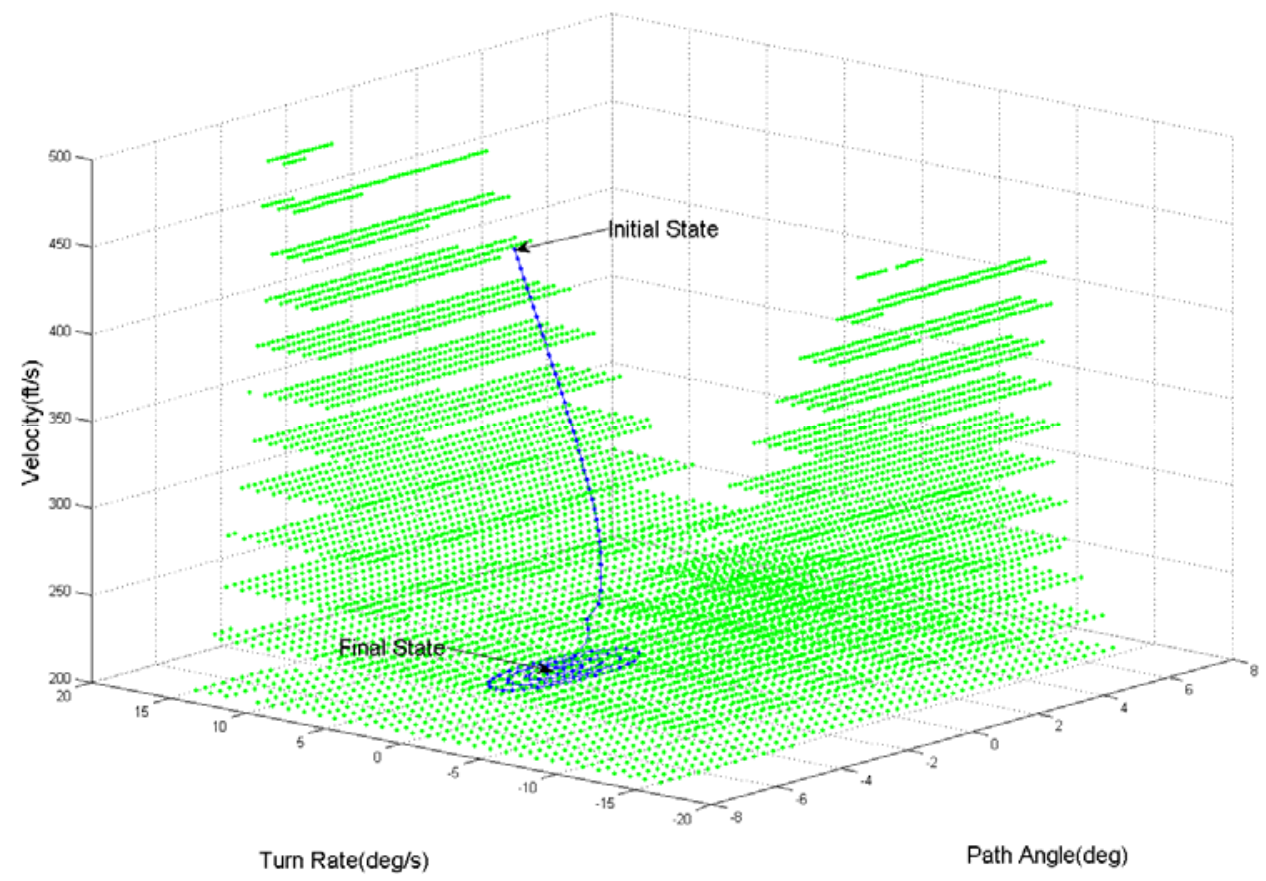

Figure 16: Trim State Discovery in 3-D Example I 


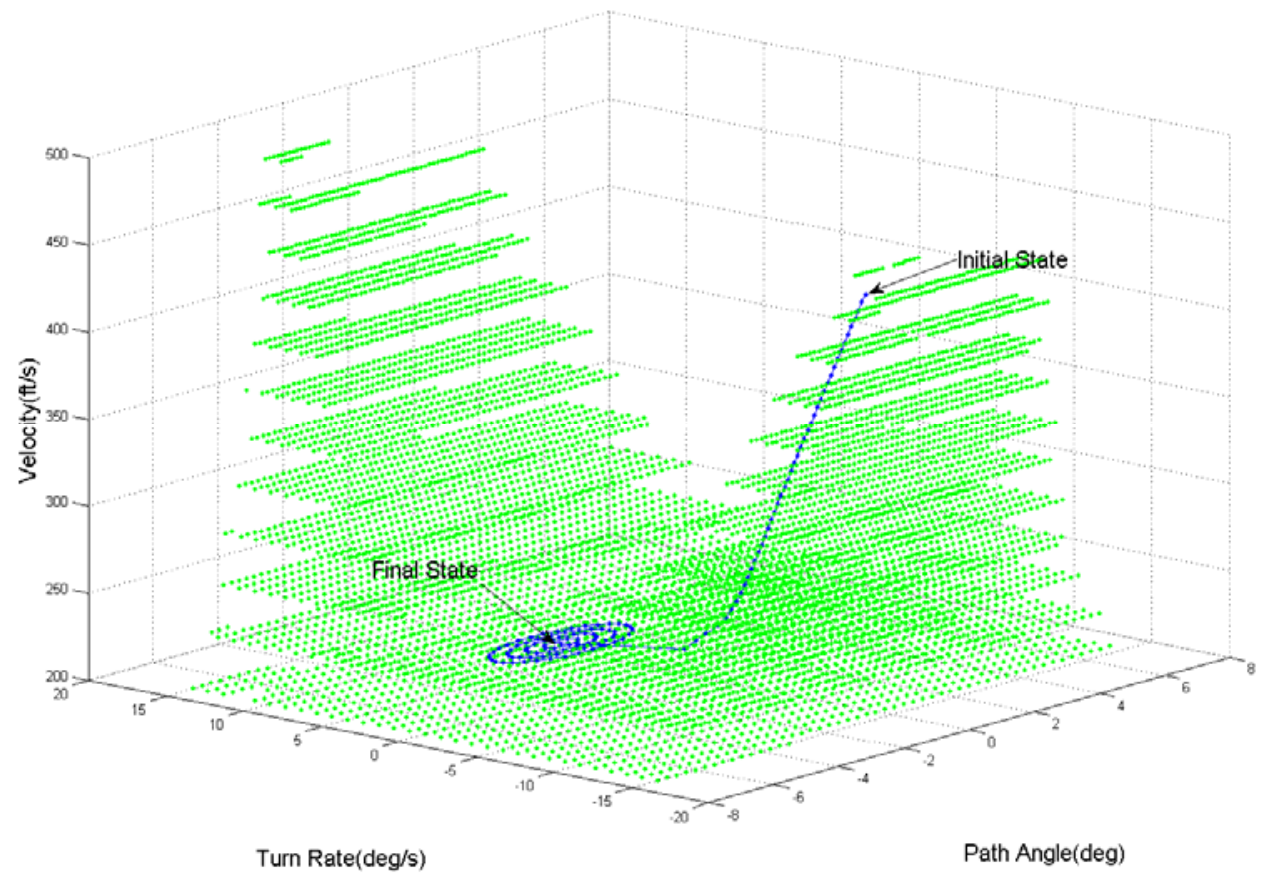

Figure 17: Trim State Discovery in 3D Example II

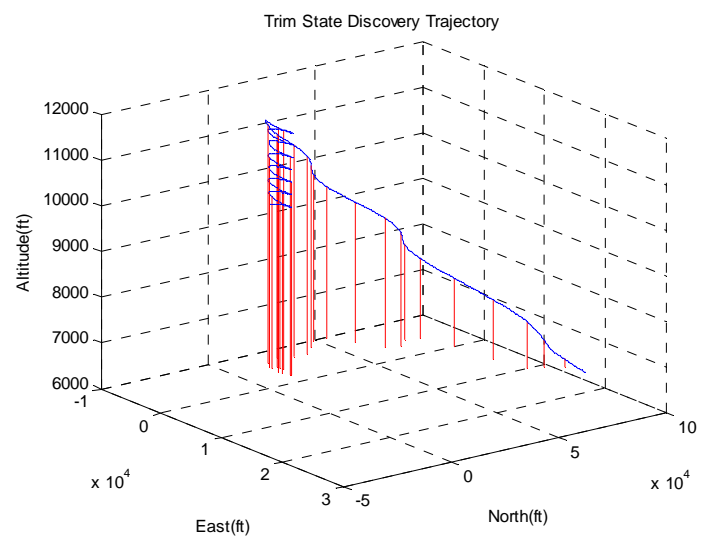

Figure 18: TSD with 5 second transitions

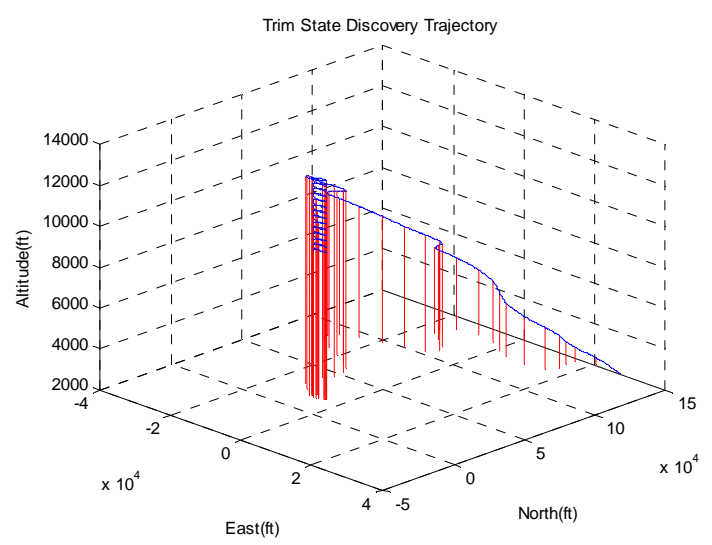

Figure 20: TSD with 20 second transitions

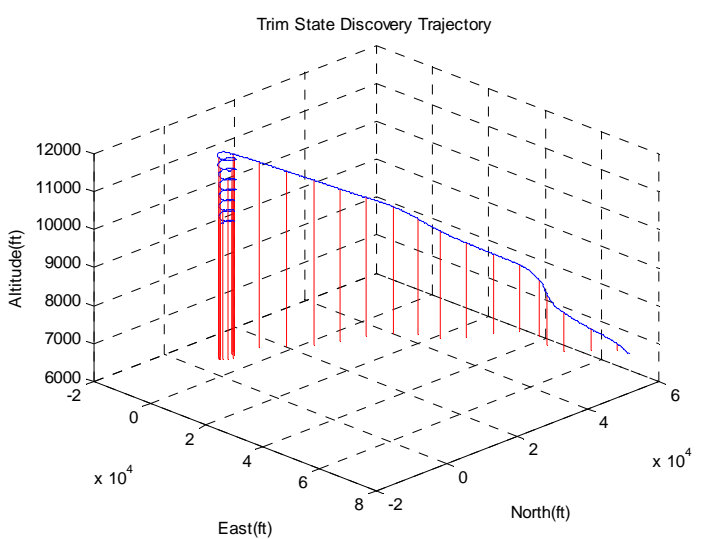

Figure 19: TSD with 10 second transitions

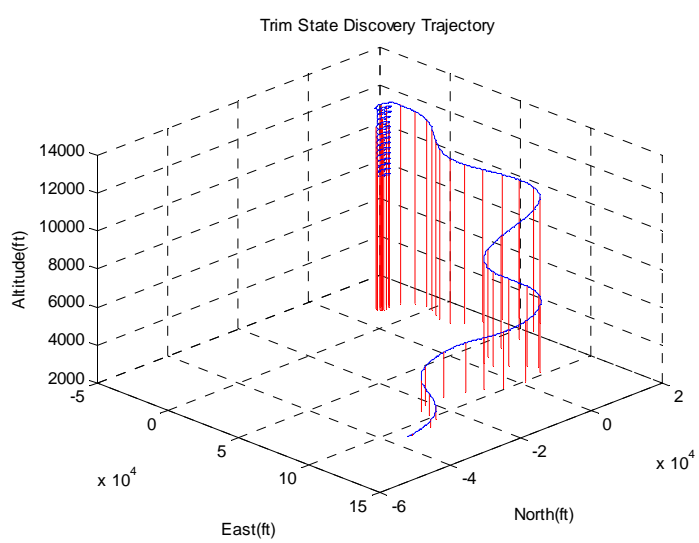

13 Figure 21: TSD with 40 second transitions

American Institute of Aeronautics and Astronautics 


\section{Conclusion}

A Trim State Discovery (TSD) algorithm has been proposed to guide a physically-disabled aircraft to explore and exploit feasible trim states that can be subsequently sequenced by an Adaptive Flight Planner (AFP) into a safe landing trajectory. TSD is of importance in situations where damage or failures cannot be explicitly characterized and matched to a known model. Emphasis is placed on efficient and correct identification of trim states at airspeed, turn rate, and flight path angle values advantageous for landing approaches. The concept of attraction region is incorporated to explore the local neighborhood of each achieved trim state to determine stability of the states in that neighborhood. An artificial potential field motion planning method has been implemented to effectively explore and exploit the target trim state neighborhood while respecting constraints. To deal with local minima, the main shortcoming of the potential field method, we proposed an edge-following method with recovery criteria. A case study with an F-16 aircraft experiencing a jammed aileron has been presented to illustrate the effectiveness of TSD. Trim State Discovery (TSD) is first demonstrated as a search over the two-dimensional plane of turn rate and path angle, expected to enable faster transitions than airspeed changes. To handle closed spaces and a more efficient (straight) path to the target approach state, the TSD algorithm was expanded to a three dimensional velocity, turn rate and path angle search-space. Once TSD is complete, all the identified trim states can be sequenced by the Segmented Trajectory Planner in the Adaptive Flight Planner (AFP) to plan a safe landing trajectory. Ideally, the AFP will use trim states around the targeted final approach trim state in a minimum-length Dubins path capable of landing the damaged aircraft. ${ }^{16}$ In the worst case, the landing trajectory can be planned in real-time to use a more restricted set of feasible trim states using our recent Turning Dubins Vehicle (TDV) method applicable to situations in which an aircraft cannot fly straight. ${ }^{33}$

Determination of $t_{\text {rans }}$ requires further study. First, what interval does an adaptive controller require to identify the Region of Attraction (RoA) presented by $t_{s h}$ ? Next, what transition time $t_{s}$ is generally optimal to transition between two neighboring trim states? Minimum $t_{s}$ enables the TSD process to conclude quickly, but too short transition time will likely produce significant overshoot, compromising the controller's ability to estimate controller parameters at the new state before it again transitions.

Trim State Discovery(TSD) establishes a feasible trim state database online in response to an unknown failure/damage event. In our case studies, the final approach trim state was feasible, but this may not always be the case. In practice, the final approach (target) trim state could be a set of stable states rather than a single point. Faced with an infeasible final trim state, an alternate target trim state must be calculated. Figure 22 shows such a situation; further work is required to determine both a procedure for altering the target trim state such that TSD can be appropriately transitioned to the landing phase in a timely manner.

In present research, the step length between trim state iterations is fixed. Optimal step length calculation should ultimately based on region of attraction size and expected proximity to envelope boundaries, since ultimately the system would transition directly to the approach state if the direct transition is feasible, although small steps within the local region of attraction are essential to ensure trim state commands never exit the degraded performance envelope.

The Adaptive Flight Planner (AFP), including with Trim State Discovery (TSD), is cast in the context of flight management to be a reference for the flight crew who can choose to accept, modify, or ignore its results. Although best practices to merge an AFP into the flight deck must still be developed, such a capability represents a new frontier in adaptivity that, once certified and adopted, can move our air transportation system beyond simply reverting control to the pilot in the most dangerous degradation scenarios.

\section{Acknowledgements}

The authors would like to thank collaborators Robert Sanner from the University of Maryland and Hee Jun Choi from the University of Michigan for their valuable insights and synergistic contributions to our adaptive flight 
planner. This research was supported in part under the NASA Aviation Safety program, Integrated Resilient Aircraft Control (IRAC) NRA Cooperative Agreement NNX08AB93A.

\section{References}

1 “Statistical summary of commercial jet airplane accidents," Research report. Boeing commercial airplanes. July 2008. http://www.boeing.com/news/techissues.

2 J. Stoop and J. Kahan, "Flyling is the safest way to travel: How aviation was a pioneer in independent accident investigation,” European Journal of Transport and Infrastructure Research, 2005, pp.115-127.

${ }^{3}$ K. W. Williams, "A summary of unmanned aerial aircraft accident/incident data: Human factors implications," Technical report. 2004.

${ }^{4}$ M. Lansdall, L. Lewis, W. J. Bezdek, “The history of commercial simulators and the Boeing 777 systems integration lab,” AIAA Modeling and Simulation Technologies Conference and Exhibit, August 2004.

${ }^{5}$ R. L. Helmreich and A. C. Merritt, "Safety and error management: The role of Crew Resource Management In B.J. Hayward \& A.R. Lowe (Eds.),” Aviation Resource Management, 2000, pp. 107-119.

${ }^{6}$ D. A. Wiegmann and S. A. Shappell, "Human error analysis of commercial aviation accidents: application of the human factors analysis and classification system (HFACS)," Aviation, Space, and Environmental Medicine, vol. 72, No. 11, 2001.

${ }^{7}$ K. W. Williams, “A Summary of Unmanned Aircraft Accident/Incident Data: Human Factors Implications,” Technical report, 2004.

${ }^{8}$ M. Bodson, J. E. Groszkiewicz, “Multivariable Adaptive Algorithms for Reconfigurable Flight Control,” IEEE Trans on Control Systems Technology, vol. 5, No. 2, 1997, pp. 217-229.

${ }^{9}$ E. N. Johnson and S. K. Kannan, "Adaptive Flight Control for an Autonomous Unmanned Helicopter," AIAA Guidance, Navigation and Control Conference, Monterey, CA, August 2002.

${ }_{10}$ M. Steinberg, "A Historical Overview of Research in Reconfigurable Flight Control," Journal of Aerospace Engineering, vol. 219, No. 4, 2005, pp. 263-275.

${ }^{11}$ R. T. Rysdyk and A. J. Calise, “Fault Tolerant Flight Control via Adaptive Neural Network Augmentation," AIAA Guidance, Navigation, and Control Conference and Exhibit, Boston, MA, Aug. 10-12, 1998, pp.1722-1728.

12 J. Kaneshige, J. Bull, J. Totah, "Generic Neural Flight Control and Autopilot System," AIAA Guidance, Navigation, and Control Conference, Denver, CO, August 2000.

${ }^{13}$ N. Nguyen, K. Krishnakumar, J. Kaneshige, "Flight dynamics and Hybrid Adaptive Control of Damaged Aircraft,” Journal of Guidance, Control, and Dynamics, vol. 31, No. 3, May-June 2008, pp. 751-759.

${ }^{14}$ A. B. Page, E. D. Meloney, J. F. Monaco, "Flight Testing of a Retrofit Reconfigurable Control Law Architecture Using an F/A-18C," AIAA Guidance, Navigation, and Control Conference and Exhibit, Keystone, Colorado, August 2006.

${ }^{15}$ T. L. Chen and A. R. Pritchett, "Development and Evaluation of a Cockpit Decision Aid for emergency Trajectory Generation,” Journal of Aircraft, vol. 38, No. 5, September-October 2001, pp. 935-943.

${ }^{16}$ E. Atkins, A. Portillo, I. Strube, "Emergency Flight Planning Applied to Total Loss of Thrust," AIAA Journal of Aircraft, vol. 43, No. 4, Jul-Aug 2006, pp. 1205-1216.

${ }^{17}$ Y. Tang, E. Atkins, R. Sanner, "Emergency Flight Planning for a Generalized Transport Aircraft with Left Wing Damage," AIAA Guidance, Navigation and Control Conference and Exhibit, Hilton Head, South Carolina, August 2007.

${ }^{18}$ M. J. Strube, “Post-failure Trajectory Planning from Feasible Trim State Sequences,” Master thesis. University of Maryland. 2005.

19 A. N. Michel, B. H. Nam, V. Vittal, "Computer Generated Lyapunov Functions of Interconnected Systems: Improve Results with Applications to Power Systems,” IEEE Trans. Circuits Syst, vol. 31, 1984, pp. 189-198.

${ }^{20}$ A. Vannelli, M. Vidyasagar, "Maximal Lyapunov Functions and Domains of Attraction for Autonomous Nolinear Systems," Automatica, vol. 21, January 1985, pp: 69-80.

${ }^{21}$ H. Chiang, M. W. Hirsch, F. F. Wu, "Stability Regions of Nonlinear Autonomous Dynamical Systems," IEEE Trans. Automatic Control, vol. 33, No. 1, January 1998.

${ }^{22}$ G. Chesi, A. Garulli, A. Tesi, A. Vicino, "LMI-based computation of optimal quadratic Lyapunov functions for odd polynomial systems," International Journal of Robust and Nonlinear Control, vol. 15, December 2004, pp. 3549.

${ }^{23}$ B. Tibken, "Estimation of the domain of attraction for polynomial systems via LMIs," Proc. 39th IEEE Conference of Electr. \& Inf. Eng., vol. 4, December 2000, pp. 3860-3864. 
${ }^{24}$ F. Amato, C. Cosentino, A. Merola, “On the Region of Attraction of Nonlinear Quadratic Systems,” Automatic, vol. 43, 2007, pp. 2119-2123.

${ }^{25}$ B. Kohout, "Challenges in real time obstacle avoidance," AAAI Spring Symposium on Real-Time Autonomous Systems, 2000.

${ }^{26} \mathrm{~V}$. Lumelsky and T. Skewis, "Incorporating range sensing in the robot navigation function," IEEE Tran. Systems Man and Cybernetics, vol. 20, 1990. pp. 1058-1068.

${ }^{27}$ V. Lumelsky, "Path-planning strategies for a point mobile automaton amidst unknown obstacles of arbitrary shape,” Algorithmic, vol. 2, 1987, pp. 403 - 430.

${ }^{28}$ O. Khatib, "Real-time obstacle avoidance for manipulators and mobile robots," International Journal of Robotics Research, vol. 5, No. 1, 1995. pp. 90-98.

${ }^{29}$ Eswar R. Reddy, Rajagopal Kuderu, Prasanna L. Kaujala, "Robots with Obstacles,” Research Journal of Applied Sciences, vol. 2, No. 12, 2007. pp. 1218-1222.

30 J. Borenstein, Y. Koren, "Histogramic in-motion mapping for mobile robot obstacle avoidance," IEEE Trans. Robotics and Automation, vol. 7, no. 4, 1991, pp. 535-539.

${ }^{31}$ Tommie Liddy, Tien-Fu Lu, "Waypoint Navigation with Position and Heading Control using Complex Vector Fields for an Ackermann Steering Autonomous Vehicle," Australasian Conference on Robotics and Automation, December 2007.

${ }^{32}$ M. Rickert, O. Brock, A. Knoll, "Balancing Exploration and Exploitation in Motion Planning," Proc. IEEE International Conference on Robotics and Automation, Pasadena, USA, May 2008, pp. 2812-2817.

${ }^{33}$ H. J. Choi and E. M. Atkins, "An Analytic Trajectory Planner for Aircraft with Severe Damage or Failures," AIAA Unmanned Unlimited Conference, Seattle, Washington, April 2009. 